\title{
A Novel Joint Chaining Graph Model for Human Pose Estimation on 2D Action Videos and Facial Pose Estimation on 3D Images
}

\author{
D.Ratna kishore \\ Asst.Prof, Department of Computer Science \& Engineering Andhrapradesh,India.
}

\author{
Dr. M. Chandra Mohan \\ Professor, Department of Computer Science \& Engineering JNTUH, Hyderabad, Telangana State, India \\ Dr.Akepogu. Ananda Rao \\ Professor in CSE, Director,Academic \& Planning JNTUA, anantapuramu, Andhrapradesh,India
}

\begin{abstract}
Human pose detection in 2D/3D images plays a vital role in a large number of applications such as gesture recognition, video surveillance and human robot interaction. Joint human pose estimation in the $2 \mathrm{D}$ motion video sequence and $3 \mathrm{D}$ facial pose estimation is the challenging issue in computer vision due to noise, large deformation, illumination and complex background. Traditional directed and undirected graphical models such as the Bayesian Markov model, conditional random field have limitations with arbitrary pose estimation in 2D/3D images using the joint probabilistic model. To overcome these issues, we introduce an ensemble chaining graph model to estimate arbitrary human poses in 2D video sequences and facial expression evaluation in 3D images. This system has three main hybrid algorithms, namely $2 \mathrm{D} / 3 \mathrm{D}$ human pose pre-processing algorithm, ensemble graph chaining segmented model on 2D/3D video sequence pose estimation and $3 \mathrm{D}$ ensemble facial expression detection algorithm. The experimental results on public benchmarks 2D/3D datasets show that our model is more efficient in solving arbitrary human pose estimation problem. Also, this model has the high true positive rate, low false detection rate compared to traditional joint human pose detection models.
\end{abstract}

Index Terms - Human Pose Estimations, Chaining graph models, Edge based Filtering.

\section{INTRODUCTION}

Human pose detection is an active research in computer vision and can be extended from facial expression detection. Most of the pose detection models aim to determine the orientation and position of body parts within the image or video sequences. Several parameters have made this detection very challenging in arbitrary pose detection applications. This challenging is due to arbitrary actions, complex background noise and the distance between camera and human actions.

Traditional models of human pose detection can be divided into 3 types i.e models related to template matching, models related to color and models related to contour detection. Models related to color are efficiently detecting a human's pose on complex actions, but the main limitation of this model is that the color histogram is not optimal in real-time surveillance actions. Also, the pose detection algorithm has better accuracy on a still action images and video sequences, a drawback of this model is that many background objects have similar colors to those of hair which gives in false detection.

The models related to template matching, detect segments belonging to head pose by computing the similarity measure of a pose template and the segmented area. Generally, pose templates are defined in terms of geometric shapes such as lines, circles, polygons, arcs and rectangle to determine the level of matching. Generally, these models are efficient to complex background and texture segmented regions, but the computational cost is too high for detection.

Models related to contour detection use some predefined geometric shapes to define body parts contour details. These models involve a constraint optimization which is efficient to noisy poses and noisy edge information. However, the curve fitting process is very time consuming process.

Predicting the 3D human pose using a template or contour is a severely under constrained problems, because many poses posses very similar projections in each direction.

The Hidden Markov model is the well known undirected graph based model for image segmentation. But, the Markov random field(MRF)[4] model incorporates the spatial relationships among the graph nodes. So, an improvement to this MRF model is a conditional random field (CRF) is also an undirected graph based model applicable for image segmentation. Traditionally, the human activity can be detected by using color based segmentation such as k-means, agglomerative and histogram approaches with edge detection algorithms. Digital image segmentation plays a significant role for partitioning an image into similar objects based on domain knowledge. Feature selection is an important 
requirement in most of the digital image segmentation techniques. Depending on the extracted features, the segmentation approaches are classified into 3 categories, namely, edge-based, the region based and thresholding based segmentations.

\section{A. Human Pose Estimation Issues and solutions}

\section{a) The Eyes brows pose estimation}

The 3D facial feature around the eyes tends noisy edges due to the reflection constraints and eye lashes. Facial texture gives high descriptive information about the shape or pose of the eye. For this reason, 3D facial features are used to find the key points around the eyes, namely inner, outer eye and around the iris region.

\section{b) Nose pose estimation}

Nose region is distinct in surface curvature, but plain in texture description. So, in order to detect the pose estimation in facial features, yaw angle of the $3 \mathrm{D}$ face is used.

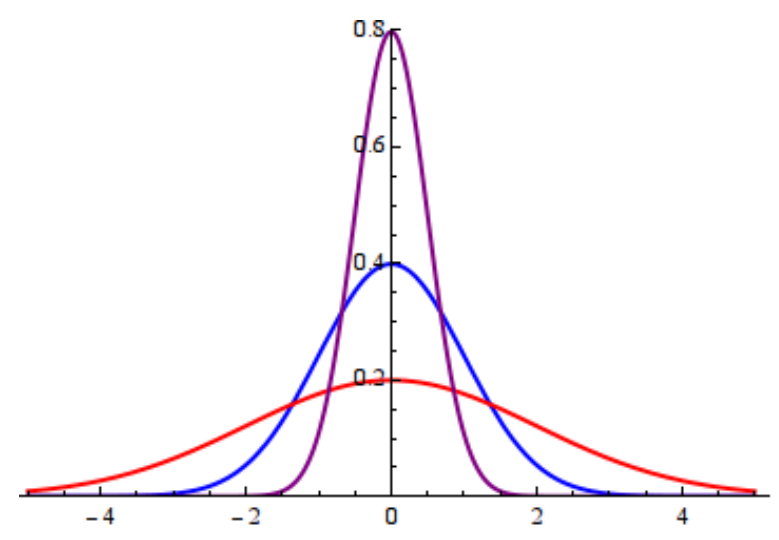

Fig.1. ROC Curves for nose error correction

Using ROC as shown in Fig. 1 , the curve is repeatedly rotated to minimize this nose difference between the two symmetrical nose regions by computing maximal point as the new nose region. Once the key point of the nose is detected this entire surface is rotated and thus nose poses are estimated accurately.

\section{c) Lips pose estimation}

As we know the color difference between the lips and skin is not always same.Due to this reason, region based contours approach is used to evaluate poses.Different facial expression are shown in the fig. 2 .

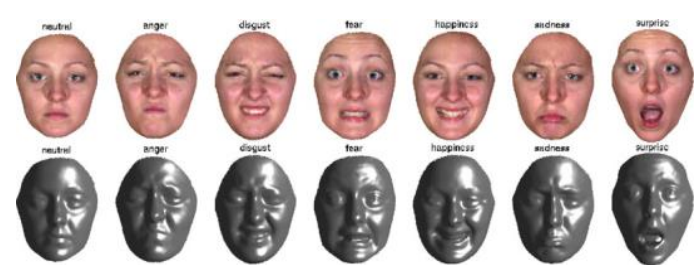

Fig.2. Facial expressions
The segmentation of human emotions or expressions are challenging factor in many real-time human activity identification. It is common in many domains such as biometric identification and medical fields which exhibit irregular boundaries and discontinuities. Also, segmented sub-regions can only be partitioned when several modalities are merged, which requires an efficient registration process in the pre-processing step. In the traditional human activity detection models, intra and inter region resolutions may affect the segmentation accuracy. Due to the partial volume effect, inherent noise, wide range of image features and spatial constraints, conventional models fail to detect the exact location and segmentation of the human activities in large training datasets.

\section{RELATED WORKS}

The traditional graph based models for image segmentation are based on either directed graphical or undirected graphical models. But, they often fail to capture the complex region relationship of different activities. In [5],color texture analysis to extract spectral correlation features is proposed. Both approaches doesn't handle texture boundary extraction due to noise in the image. A more improved approach is proposed in the Texem model, which consists of a conditional dependency between neighbor regions and it is totally based on Gaussian mixture model. Digital image thresholding is one the most popular method due to its simple implementation and intuitive features. Consequently, the threshold measures for multimodal histograms must be minima among the two maxima. A few techniques enlighten the histogram peaks in image feature extraction stage so as to provide the threshold detection. The main drawback of this method is to segregate the object from background if the object and background regions are identical pixel distribution. Edge based segmentation works well against irregularities in image attributes such as texture, gray level, color etc. These irregularities are known as edges and are noticed using edge detection operations, some of the generally used operations are prewitt, Laplace, sobel etc. Segmentation using edge-based method could be used as incomplete due to the occurrence of stray, broken or noisy edges. Enhanced image processing is essential to extract the edges corresponding to rational objects. Several edge based methods have been proposed in the literature, but the frequently accepted segmentation systems are edge based thresholding, which is used to clear noisy edges in bright conditions. Edge image thresholding directs to stray edges in the presence of noise where the actual edges are frequently missing [6][7] Stray edge problems can be cleared if the edge properties are decided with respect to the mutual neighbor, while the presence of edge based on the strength of edges in the near neighborhood. Region based segmentation approach which depends on the homogeneity measure to divide and merge regions in an image so as to broaden semantic or useful division in the processed image. 


\section{A. Conventional Human Activity Detection Methods}

The region growing model is proposed in $\mathrm{C}-\mathrm{V}$ segmentation. For a given frame I on the domain, C represents the closed curve region and $\mathrm{i} 1$ and $\mathrm{i} 2$ represents the average inside and outside intensities of the closed curve $\mathrm{C}$. The basic assumption of this segmentation by optimizing the energy function as:

$$
\begin{aligned}
E(i 1, i 2, C)= & \mu_{X} \cdot \operatorname{len}(C)+\omega \cdot \operatorname{area}(C) \\
& +\theta_{1} \int_{i n s(C)}|I-v 1|^{2} d x d y+\theta_{2} \int_{i n s(C)}|I-v 2|^{2} d x d y
\end{aligned}
$$

Where $\mu_{X}>0, \omega>0, \theta_{1}, \theta_{2}>0$ are fixed values.

The partial differentiation equation corresponding to the given $\mathrm{C}-\mathrm{V}$ model is evaluated as

$$
\begin{aligned}
\partial \varphi / \partial t & =\frac{d f(x)}{d x}\left[\mu_{x} \operatorname{div}(\nabla \varphi /|\nabla \varphi|)-\omega-\theta_{1}(I-v 1)^{2}+\theta_{2}(I-v 2)^{2}\right] \\
& \text { where } \mathrm{v} 1=\int_{\Omega} \mathrm{I} . \mathrm{f}(\mathrm{x}, \mathrm{y}) \mathrm{dx} \cdot \mathrm{dy} / \int_{\Omega} \mathrm{f}(\mathrm{x}, \mathrm{y}) \mathrm{dx} \cdot \mathrm{dy} \\
& \mathrm{v} 2=\int_{\Omega} \mathrm{I} .(1-\mathrm{f}(\mathrm{x}, \mathrm{y})) \mathrm{dx} \cdot \mathrm{dy} / \int_{\Omega}(1-\mathrm{f}(\mathrm{x}, \mathrm{y})) \mathrm{dx} \cdot \mathrm{dy} \\
& \mathrm{f}(\mathrm{x})=1, \text { if } \mathrm{x} \geq 0 \\
& \mathrm{f}(\mathrm{x})=0, \text { if } \mathrm{x}<0
\end{aligned}
$$

This model assumes that the image intensity values in each adjacent block always uniform.

Self Organizing Map (SOM), as part of competitive learning neural network technique has been used to develop the vector quantization process. The role of SOM for vector quantization process is mainly due to the pixel similarity between the region learning applied in the self organizing map. Neural units in the competitive layer need to be nearly equal to the number of regions specified in the segmented image. This is the main disadvantage of traditional SOM for image segmentation.

An optimization to $\mathrm{C}-\mathrm{V}$ model was implemented by Zhang et al[8] which consider intensity data to find the refined edges. Thus, using gradient information, the gradient based $\mathrm{C}-\mathrm{V}$ model is proposed. The basic optimization in GCV model is:

$$
\begin{aligned}
& E(v 1, v 2, d s 1, d s 2, \eta)=\mu_{X} \int_{\Omega} 0.5(|\nabla \eta|-1)^{2} d x d y+ \\
& \left.\left.+\theta_{1} \int_{\text {ins }(C)} \mid I(x, y)-v 1\right)\left.\right|^{2} d x \cdot d y+\theta_{2} \int_{\text {ins }(C)} \mid I(x, y)-v 2\right)\left.\right|^{2} d x \cdot d y+ \\
& \left.\left.\int_{\Omega \text { inss }(C)} \int_{\text {inss }(C)} \mid I(x, y)-v 1\right)\left.\right|^{2} d x \cdot d y+\theta_{2} \int_{\mid} \mid I(x, y)-v 2\right)\left.\right|^{2} d x . d y
\end{aligned}
$$

\section{B. Problems in Traditional Human activity segmentation Algorithms}

- Conventional Segmentation algorithms fail to differentiate the human activities with the corner edges.

- Each activity depends on the histogram and user specified threshold. Need to specify a dynamic or global and local threshold.
- Fails to identify the symmetrical activities due to noisy pixels in the central region.

- Fails to detect new types of activities in the test data.

- Difficult to segment the human expressions using the activity list.

\section{Traditional Joint Bayesian Probabilistic Chain Graphical Model}

Each probabilistic graph model consists of two phases: quantitative and qualitative parts. Each probabilistic graph model is denoted by a graph $\mathrm{G}=(\mathrm{V}, \mathrm{E})$; where $\mathrm{V}$ is the set of vertices and $\mathrm{E}$ is the edge set as shown in Fig.3.

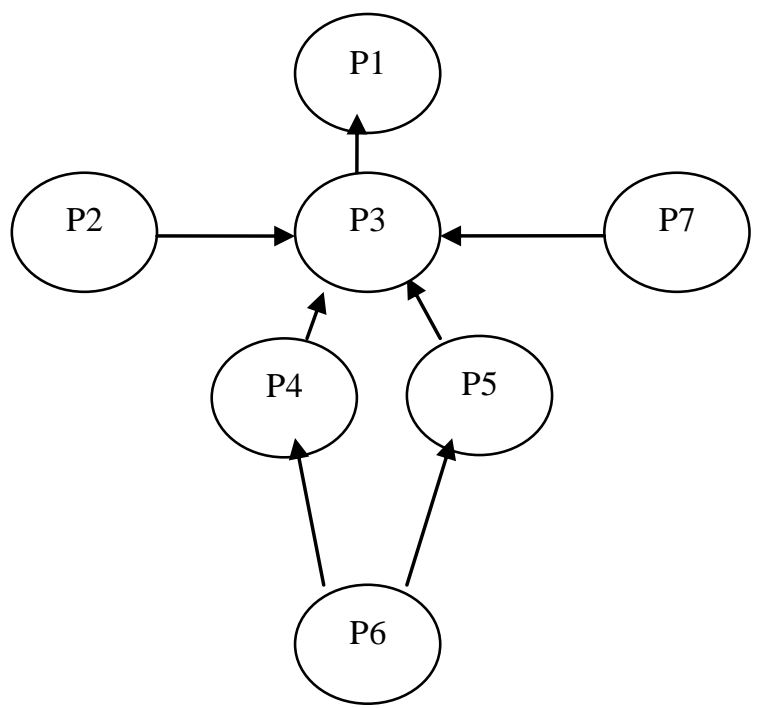

Fig.3. Bayesian directed chaining graph model

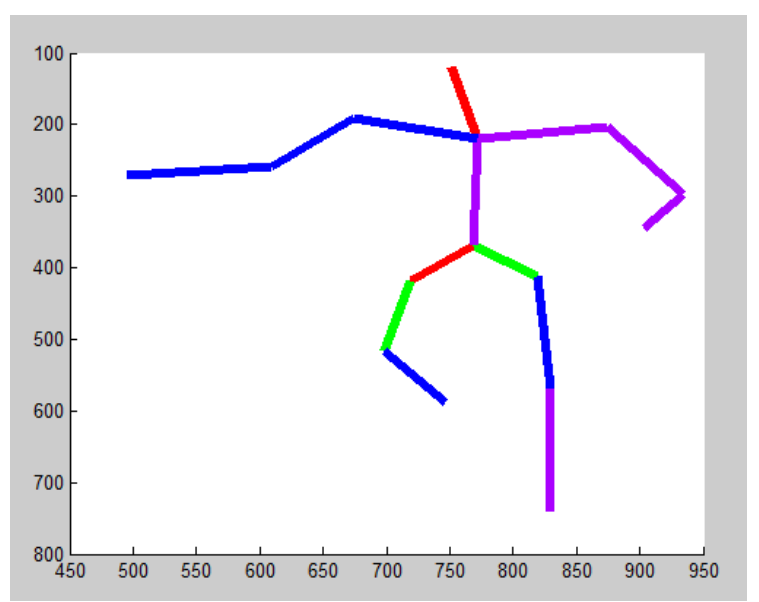

Fig.4. Corresponding 2D action estimation to chaining graph model

Each node in the vertices set represents the random variable and the link or edge represents the prior probabilistic relationships among the node random variables. In the novel chain graphical model, graph contains both directed and undirected edges. Each edge in the novel chain graphical model represents the joint probability distribution among the random variables. Figure 3 shows the Bayesian chaining graph model for pose detection on 2D images. Probabilistic Bayesian 
model is used to predict the discrimination evidence and to estimate pose from these evidences as shown in Fig.4.

Let $\mathrm{L}_{\mathrm{i}}=\left(\mathrm{x}_{\mathrm{i}}, \mathrm{y}_{\mathrm{i}}, \mathrm{s}_{\mathrm{i}}\right) \cdot\left(\mathrm{x}_{\mathrm{i}}, \mathrm{y}_{\mathrm{i}}\right)$ is the pose position with respect to centre of the action image and $s_{i}$ is the scaling factor. $D=\left\{d_{1}, d_{2} \ldots d_{N}\right\}$ be the image feature points for the whole action image. Then, the likelihood estimation to the given specific pose in the image evidence is given by:

$$
\operatorname{Prob}(\mathrm{D} / \mathrm{L})=\prod_{\mathrm{I}=1}^{\mathrm{N}} \operatorname{Prob}\left(\mathrm{D}=\left\{\mathrm{d}_{1}, \mathrm{~d}_{2} \ldots \mathrm{d}_{\mathrm{N}}\right\} / \mathrm{L}_{\mathrm{i}}=\left(\mathrm{x}_{\mathrm{i}}, \mathrm{y}_{\mathrm{i}}, \mathrm{s}_{\mathrm{i}}\right) .\left(\mathrm{x}_{\mathrm{i}}, \mathrm{y}_{\mathrm{i}}\right)\right)
$$

The pose estimation problem is to detect the orientation and location of a 3D object through object mapping in between the $3 \mathrm{D}$ object to the $2 \mathrm{D}$ pose. The Gaussian energy function for pose scanning can be estimated using the following function.

$$
\begin{gathered}
g(x)=e^{-\left(\mu_{2} / 4 \sigma_{1}^{2}\right)\left(x-\mu_{1}(x)\right)^{2}(f(x, y)-0.5)} \\
\text { if } y=m(\text { row size of the block or pose) } \\
g(x)=e^{-\left(\mu_{2} / 4 \sigma_{2}^{2}\right)\left(x-\mu_{2}(x)\right)^{2}(f(x, y)-0.5)} \\
\text { if } y=n(\text { column size of the block or pose) }
\end{gathered}
$$

\section{Where}

$\mu_{1}$ and $\mu_{2}$ are the pose mean; $\sigma_{1}^{2}$ and $\sigma_{2}^{2}$ are the pose variance.

In this model, to classify for a particular pose class, using the Bayesian classifiers the entire pose class set is a computational overhead. In this joint boosting model, candidate pose set is pre-selected at random from the training data. During training phase, image background is trained into a class that discriminates pose from the background. This model is used to classify multi-pose learning that is used in image classification and key-pose extraction.

\section{3D-Facial pose estimation}

[5] Implemented the enhanced PCA-ICA scheme to estimate facial features recognition using only the texture description on the predefined poses training data. They extracted the face pose contours in the given query image around the eyes, eyebrows, nose and mouth by implementing an active contour techniques. These pose contours were mapped onto the 3D-mesh data to generate the texture information subjected to the enhanced PCA model to recognize expressions.

In general, most of the literature work on 3D-facial expression evaluation can be classified as feature based classification or generic facial based classification. In the first category, 3D facial poses are scanned and classify them into various facial expressions. Most of these models, the $3 \mathrm{D}$ face is sub partitioned into regions using manually annotated landmarks, principal direction and surface curvature to recognize various facial expressions.
In the second category, general facial features are constructed using dense correspondence on a set of training 3D feature points[12-15].

The facial pose estimation between each frame in the 3D facial training data are used to annotate pose regions using the B-spline interpolation. Let $\mathrm{F} 1=(\mathrm{x} 1, \mathrm{y} 1, \mathrm{z} 1)$ and $\mathrm{F} 2=(\mathrm{x} 2, \mathrm{y} 2, \mathrm{z} 3)$ are the two facial pose key features used to estimate pose vector field $\mathrm{P}(\mathrm{F})$ such that,

$$
\mathrm{F} 2=\mathrm{P}(\mathrm{F})+\mathrm{F} 1 ;
$$

Let $\mathrm{L}(\phi)$ be the lattice structure of the regular source image which consists of $n(x) \times n(y) \times n(z)$ feature points in each direction with regular space field $\phi(\mathrm{i}, \mathrm{j}, \mathrm{k})$. The pose transformation of the B-spline interpolation of the 60 closest feature control points is given by

$$
\mathrm{P}(\mathrm{F})=\sum_{\mathrm{i}=0}^{3} \sum_{\mathrm{j}=0}^{3} \sum_{\mathrm{k}}^{3} \mathrm{~B}_{\mathrm{x} 1, \mathrm{x} 2, \mathrm{x} 3}(\mathrm{l}) \mathrm{D}(\mathrm{i}, \mathrm{j}, \mathrm{k})
$$

Where $\quad \mathrm{x} 1=\mathrm{x}-\mathrm{x}_{0}, \mathrm{x} 2=\mathrm{y}-\mathrm{y}_{0}, \mathrm{x} 3=\mathrm{z}-\mathrm{z}_{0} ; \quad$ and $\left(\mathrm{x}_{0}, \mathrm{y}_{0}, \mathrm{z}_{0}\right)$ is the initial pose point.

$\mathrm{B}_{\mathrm{x} 1, \mathrm{x} 2, \mathrm{x} 3}(\mathrm{l})$ is the basis function of cubic $\mathrm{B}$ Spline ,defined as

$$
\begin{aligned}
& \mathrm{B}\left(1_{0}\right)=(1 / 6)\left(-\mathrm{x}^{3}+3 \mathrm{x}^{2}-3 \mathrm{x}+1\right) \\
& \mathrm{B}\left(1_{1}\right)=(1 / 6)\left(3 \mathrm{x}^{3}+6 \mathrm{x}^{2}+4\right) \\
& \mathrm{B}\left(1_{2}\right)=(1 / 8)\left(-3 \mathrm{x}^{3}+3 \mathrm{x}^{2}+3 \mathrm{x}+1\right) \\
& \left.\mathrm{B}\left(1_{3}\right)=1 / 6\right)\left(\mathrm{x}^{3}\right)
\end{aligned}
$$

$\mathrm{P}(\mathrm{F})$ is the facial expression evaluation function.

\section{PROPOSED MODEL}

\section{A. Probabilistic based Human Pose Detection in 2D Action Video Sequences}

In this proposed model, 2D video action sequences are used to detect the accuracy human poses using enhanced chaining graph model with filtering algorithm. The conditional dependence represents the relationship of the occurrence of one object being dependent on the occurrence of the other object and asymmetric in nature. Also, the homogeneous association usually computes the similarity among the objects. The main aim of the chaining graph segmentation algorithm is to partition an image into homogeneous regions (classes) with respect to one or more pose characteristics. Proposed segmentation algorithm is based on probabilistic estimation of the human action and non-actions regions as shown in Fig. 5. In this human pose detection algorithm, the basic chain graph algorithm is applied on the pre-processed image for initial segments. Initial segments have a large number of over segmented regions which are very difficult to detect or process the activities in the image. 


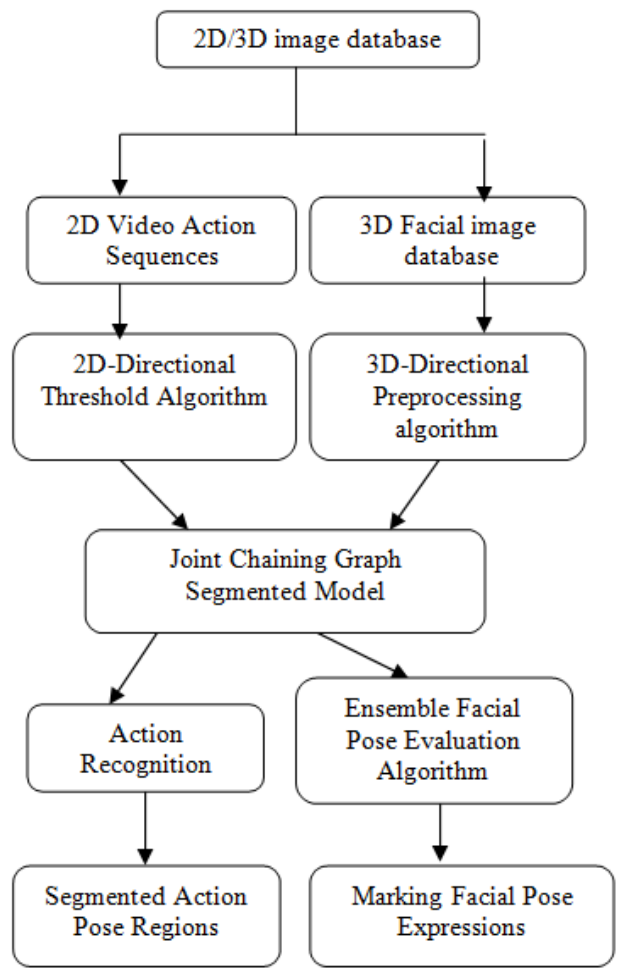

Fig.5. Proposed Joint Human Pose Detection Model

In order to overcome this issue, the probabilistic estimation method is applied in the over-segmented regions to extract the activities by merging the estimated regions. In this proposed model, 2D/3D data are used to find the pose estimation in $2 \mathrm{D}$ and $3 \mathrm{D}$ facial expressions. The proposed model is summarized in the Figure 5. In this model two phases are used to detect the poses. In the first phase, 2D video sequences are used to find the action poses using the 2D directional filtering algorithm and chaining graph segmentation model. Directional filtering algorithm is used to filter the noise in each direction $\mathrm{x}$ axis and $y$-axis and enhanced chain graph model is used to find the pose actions using the foreground and background segmented regions. In the second phase, 3D facial database are used to evaluate the $3 \mathrm{D}$ facial expressions using 3D facial pre-processing algorithm, joint segmentation algorithm and the ensemble facial expression evaluation algorithm. Algorithm 1 and 2 describe the 2D video sequences pre-processing algorithm and the chaining graph segmentation algorithms for pose detection in the video sequences. Algorithm 3 and 4 describe the 3D facial filtering algorithm and the ensemble segmented model for facial expression evaluation algorithm on the $3 \mathrm{D}$ facial database.

Algorithm 1: 2D Video Sequence Pre-processing Algorithm

Input: Video Sequence Images

Output: Filtered Video Sequences

Procedure:

Step 1:

For each frame $\mathrm{F}$

do
Divide the human action frame into two classes $C l s_{1}$ in $\mathrm{X}$-axis and $\mathrm{Cls} s_{2}$ in $\mathrm{Y}$-axis directions with foreground level fg, background level bg and the block mean value Th such that

$$
f g=\{0,1,2 \ldots T h\}
$$

and

$$
\mathrm{bg}=\{\mathrm{Th}+1, \mathrm{Th}+2 \ldots \mathrm{Th}+\mathrm{N}\}
$$

where $\mathrm{N}$ is the total number of levels in each direction

$$
\left.\left.\operatorname{Th}=\min \left\{\sum \mathrm{f}_{\mathrm{xy}}(\mathrm{x}, \mathrm{y})\right) /\left|\mathrm{f}_{\mathrm{x}}(\mathrm{x}, \mathrm{y})\right|, \sum \mathrm{f}_{\mathrm{xy}}(\mathrm{x}, \mathrm{y})\right) /\left|\mathrm{f}_{\mathrm{y}}(\mathrm{x}, \mathrm{y})\right|\right\}
$$

Step 2: Determine the foreground level lower threshold using C-V model $T_{1}$.

The variance of foreground level $f l=\{0,1,2 \ldots T h\}$ is

$$
\begin{aligned}
\partial \varphi / \partial \mathrm{t}= & \frac{\mathrm{df}(\mathrm{x}, \mathrm{y})}{\mathrm{dx}}\left[\mu_{\mathrm{x}} \operatorname{div}(\nabla \varphi /|\nabla \varphi|)-\omega-\theta_{1}(\mathrm{I}-\mathrm{v} 1)^{2}+\theta_{2}(\mathrm{I}-\mathrm{v} 2)^{2}\right] \mathrm{s} \\
& +\frac{\mathrm{df}(\mathrm{x}, \mathrm{y})}{\mathrm{dy}}\left[\mu_{\mathrm{Y}} \operatorname{div}(\nabla \varphi /|\nabla \varphi|)-\omega-\theta_{1}(\mathrm{I}-\mathrm{v} 1)^{2}+\theta_{2}(\mathrm{I}-\mathrm{v} 2)^{2}\right] \mathrm{s}
\end{aligned}
$$

Where

$$
\begin{aligned}
& \mathrm{v} 1=\int_{\Omega} \mathrm{f}_{\mathrm{x}}(\mathrm{x}, \mathrm{y}) \mathrm{dx} / \int_{\Omega} \mathrm{f}_{\mathrm{y}}(\mathrm{x}, \mathrm{y}) \mathrm{dy} \\
& \mathrm{v} 2=\int_{\Omega} \mathrm{f}_{\mathrm{xy}}(\mathrm{x}, \mathrm{y}) \mathrm{dxdy} /\left(\int_{\Omega} \mathrm{f}_{\mathrm{x}}(\mathrm{x}, \mathrm{y}) \mathrm{dx} . \mathrm{f}_{\mathrm{y}}(\mathrm{x}, \mathrm{y}) \mathrm{dy}\right) \\
& T_{1}=\partial \varphi / \partial t * \sum\left(\mathrm{x} * \operatorname{prob}^{2}(x)-x^{2} * p r o b\right) /|N|
\end{aligned}
$$

Step 3:

Determine upper threshold $T_{2}$.

$$
T_{2}=\sum_{x=T h+1}^{N-1} \operatorname{prob}(x) * \sum_{x=T h+1}^{N-1}\left(\mathrm{x}^{*} \operatorname{prob}^{2}(x)-x^{2} * \operatorname{prob}(\mathrm{x})\right) /|\mathrm{N}|
$$

Step 4:

Check the foreground and background noise using the following condition:

$$
\begin{aligned}
& \text { if }\left(\mathrm{T}_{1}>\lambda_{1}\right) \\
& \text { remove gaussian noise(fg); } \\
& \text { else if }\left(\mathrm{T}_{2}>\lambda_{2}\right) \\
& \text { remove guassian noise(bg); } \\
& \text { else } \\
& \text { continue; }
\end{aligned}
$$

Step 6: Repeat steps 2 to 4 until the video sequence is empty.

After enhancing the given video sequences using a preprocessing algorithm, the next step is to find the human 
pose in the enhance image using proposed chaining graph model.

Algorithm 2:Optimized Region Merging Activity Segmentation Algorithm

Input:

Filtered Dimensional Data.

$e(x)=$ edgeset values along with probabilistic values of first region.

$e(\mathrm{y})=$ edge set values along with probabilistic values of first region.

$p(x)=$ intensity values of first region.

$p(y)=$ intensity values of second region.

Output : Enhanced Human Activity Segments.

Procedure:

Segmented regions $S_{i}$;

Human activites estimated over segmented Regions $S_{i}$ generated from chain graphical model.

Segmented_Regions[]=ChainGraphModel(data);

For each region reg in Segmented_Regions

Do

If(reg is oversegmented)

Then

$S_{i}=$ reg;

End if

done

Set $i=0$.

For each pair of adjacent oversegmented activities in $S_{i}$ do

Calculate the similarity between the oversegmented activities $\left(\mathrm{O}_{\mathrm{i}}, \mathrm{O}_{\mathrm{j}}\right)$ along with the area as

$$
L(i, j)=(1 / 2 \pi) * \mathrm{e}^{-\left|O_{i}-O_{j}\right|^{2} / 2^{*} \max \left\{\sigma_{i}^{2}, \sigma_{j}^{2}\right\}}
$$

Done

For each pair of nearest neighbor over-segmented activites

Do

$\mathrm{R}(\mathrm{A})=\operatorname{Region} \mathrm{Area}(\mathrm{A}), \mathrm{R}(\mathrm{B})=\operatorname{Region} \mathrm{Area}(\mathrm{B})$.

Calculate mean similarity value of all the oversegmented activities in List $\mathrm{L}$ as $\theta$

$$
\theta=L(i, j) / \mathrm{N}
$$

Where $\mathrm{N}$ is the number of over segmented regions.

If( $(\mathrm{R}(\mathrm{A}) \| \mathrm{R}(\mathrm{B}))<\theta)$

Then

$$
\begin{aligned}
& \eta=\operatorname{prob}(p(x) / p(\mathrm{y})) \\
& \eta=\frac{1}{(2 \pi)^{N_{1}} *|\sqrt{\operatorname{cov}(\mathrm{p}(\mathrm{x}), \mathrm{p}(\mathrm{y})}|} * \mathrm{e}^{\left.-(1 / 2) * N_{2}\right)}
\end{aligned}
$$

The variance of edge sets $\mathrm{x}$ is evaluated as :

$$
\begin{aligned}
& \partial e(x) / \partial t=\frac{d f(x)}{d x}\left[\mu_{e(x)} \operatorname{div}(\nabla \varphi /|\nabla \varphi|)-\omega-\theta_{1}(I-v 1)^{2}\right. \\
& \left.+\theta_{2}(I-v 2)^{2}\right]
\end{aligned}
$$

Where

$$
\begin{aligned}
& \begin{array}{r}
\mathrm{v} 1=\int_{\Omega} \mathrm{f}_{\mathrm{x}}(\mathrm{x}, \mathrm{y}) \mathrm{dx} / \int_{\Omega} \mathrm{f}_{\mathrm{y}}(\mathrm{x}, \mathrm{y}) \mathrm{dy} \\
\mathrm{v} 2=\int_{\Omega} \mathrm{f}_{\mathrm{xy}}(\mathrm{x}, \mathrm{y}) \mathrm{dxdy} /\left(\int_{\Omega} \mathrm{f}_{\mathrm{x}}(\mathrm{x}, \mathrm{y}) \mathrm{dx} \cdot \mathrm{f}_{\mathrm{y}}(\mathrm{x}, \mathrm{y}) \mathrm{dy}\right) \\
f(x)=1 \text { if } e(x) \geq 0 \\
=0 \text { if } e(x)<0
\end{array} \\
& \mathrm{e}_{x}^{\prime}=\operatorname{Max}\left\{\partial e(x) / \partial t,\left|E \cap\left\{p_{x}\right\}\right| /\left|\left\{\mathrm{p}_{x}\right\}\right|\right\}
\end{aligned}
$$

The variance of edge sets y is evaluated as :

$$
\begin{aligned}
& \partial e(y) / \partial t=\frac{d f(x)}{d x}\left[\mu_{e(y)} \operatorname{div}(\nabla \varphi /|\nabla \varphi|)-\omega-\theta_{1}(I-v 1)^{2}\right. \\
& \left.+\theta_{2}(I-v 2)^{2}\right]
\end{aligned}
$$

Where

$$
\begin{aligned}
& \mathrm{v} 1=\int_{\Omega} \mathrm{f}_{\mathrm{x}}(\mathrm{x}, \mathrm{y}) \mathrm{dx} / \int_{\Omega} \mathrm{f}_{\mathrm{y}}(\mathrm{x}, \mathrm{y}) \mathrm{dy} \\
& \mathrm{v} 2=\int_{\Omega} \mathrm{f}_{\mathrm{xy}}(\mathrm{x}, \mathrm{y}) \mathrm{dxdy} /\left(\int_{\Omega} \mathrm{f}_{\mathrm{x}}(\mathrm{x}, \mathrm{y}) \mathrm{dx} \cdot \mathrm{f}_{\mathrm{y}}(\mathrm{x}, \mathrm{y}) \mathrm{dy}\right) \\
& f(x)=1 \text { if } e(y) \geq 0 \\
& =0 \text { if } e(y)<0
\end{aligned}
$$

and

$$
\begin{gathered}
\mathrm{e}_{y}^{\prime}=\operatorname{Max}\left\{\partial e(\mathrm{y}) / \partial t,\left|E \cap\left\{p_{y}\right\}\right| /\left|\left\{\mathrm{p}_{y}\right\}\right|\right\} \\
\lambda=\left|E \cap\left\{\mathrm{e}_{x} \cup e_{y}\right\}\right| /\left(\min \left(\mathrm{e}_{x}^{\prime}, \mathrm{e}_{y}^{\prime}\right)^{*}\left|\left\{\mathrm{e}_{x} \cup e_{y}\right\}\right|\right.
\end{gathered}
$$

Where

End if

If $(\lambda<=\eta)$

Then

Merge $(p(x), p(y))$

Else

Break.

End if

done 
Algorithm 3:3D-Facial Pre-processing Algorithm Input : Yaw(Y),Pitch(P) and Roll, T(Y,P,R)

Output: 3D- Facial pre-processing image

Procedure:

Step 1: Initialize 3D image database

Step 2: Applying directional noise filtering procedure as shown below

$$
\begin{aligned}
& \mathrm{G}_{\mathrm{Y}}(\mathrm{P}, \mathrm{R})=1 / \sqrt{2 \pi \sigma_{\mathrm{Y}}} \cdot \exp \left(-0.5\left(\mathrm{x}_{\mathrm{p}}{ }^{2}+\mathrm{y}_{\mathrm{R}}^{2}\right)---(1)\right. \\
& \mathrm{G}_{\mathrm{P}}(\mathrm{Y}, \mathrm{R})=1 / \sqrt{2 \pi \sigma_{\mathrm{P}}} \cdot \exp \left(-0.5\left(\mathrm{x}_{\mathrm{Y}}{ }^{2}+\mathrm{y}_{\mathrm{R}}^{2}\right)----(2)\right. \\
& \mathrm{G}_{\mathrm{R}}(\mathrm{Y}, \mathrm{P})=1 / \sqrt{2 \pi \sigma_{\mathrm{R}}} \cdot \exp \left(-0.5\left(\mathrm{x}_{\mathrm{Y}}{ }^{2}+\mathrm{y}_{\mathrm{P}}^{2}\right)----(3)\right.
\end{aligned}
$$

Remove Gaussian noise in each direction using $G_{Y}(P, R), G_{P}(P, R)$ and $G_{R}(P, R)$.

Step 3: For each Pose $T(Y, P, R)$ do

$\alpha, \beta, \gamma$ are the 3 directional threshold limits.

If $\left(G_{Y}(P, R)>\alpha\right)$

Then

$$
\text { Guassian_remove }\left(\mathrm{G}_{\mathrm{Y}}(\mathrm{P}, \mathrm{R})\right) \text {; }
$$

Else if $\left(G_{P}(Y, R)>\beta\right)$

Then

Guassian_remove $\left(\mathrm{G}_{\mathrm{P}}(\mathrm{Y}, \mathrm{R})\right)$;

Else if $\left(G_{R}(Y, P)>\gamma\right)$

End for.

Guassian_remove $\left(\mathrm{G}_{\mathrm{R}}(\mathrm{Y}, \mathrm{P})\right)$;

Step 4: T' $(Y, P, R)=T(Y, P, R)$

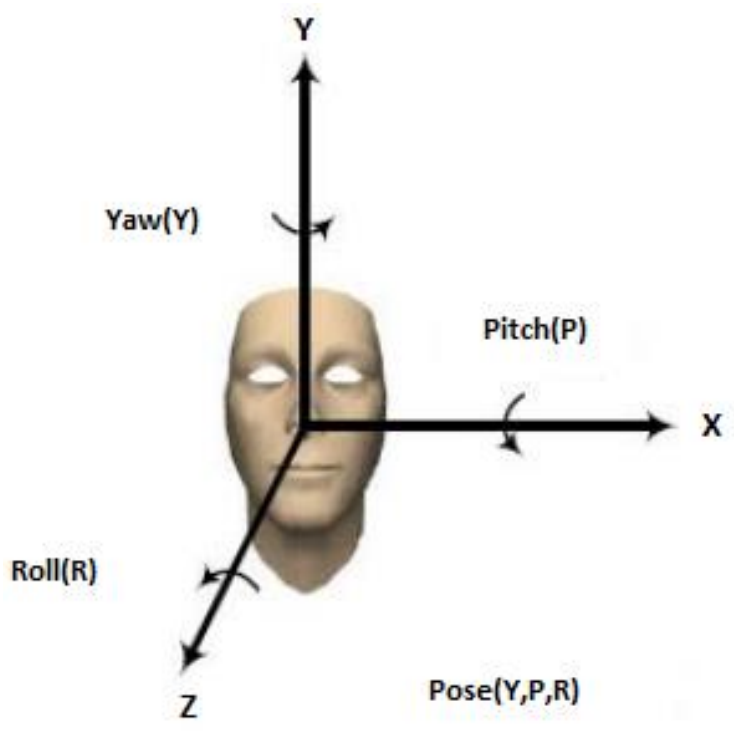

Fig.6. 3-Dimensional Face Coordinates

3D-Directional Preprocessing

This filtering model is implemented in two phases. In the first phase, holes are fixed in each direction, i.e Y,P,R direction as shown in Fig. 6. This process is used to filter the noise or ridges in each direction using $\mathrm{Knn}(\mathrm{K}$ nearest neighbor) method. In this method, Knn is applied on the $\mathrm{Y}, \mathrm{P}$ direction and then average of these k-points are set to $\mathrm{Z}$-axis ( $\mathrm{R}$ direction). These $\mathrm{k}$ points $(\mathrm{Y}, \mathrm{P})$ and $\mathrm{R}$ average points are used to fill the holes or ridges in each direction for normalization process. In the second phase, 3-D facial landmarks in each direction $\mathrm{Y}, \mathrm{P}, \mathrm{R}$ are computed using the surface ' $S$ ' representation by twice differentiation of the real valued function. So, for each point in each direction Y,P,R compute normalized mean and normalized curvatures as follows:

$$
\begin{gathered}
\mathrm{NM}(\text { Normalized-Mean })=\frac{\left(1+\mathrm{f}_{\mathrm{xy}}^{2}\right) \mathrm{f}_{\mathrm{yy}}-2 \mathrm{f}_{\mathrm{x}} \mathrm{f}_{\mathrm{y}} \mathrm{f}_{\mathrm{xy}}+\left(1+\mathrm{f}_{\mathrm{y}}^{2}\right) \mathrm{f}_{\mathrm{xx}}}{2\left(1+\mathrm{f}_{\mathrm{xy}}^{2}+\mathrm{f}_{\mathrm{x}}^{2}+\mathrm{f}_{\mathrm{y}}^{2}\right)} \\
\mathrm{NM}(\text { Normalized-Mean })=\frac{\left(1+\frac{\partial^{2} \mathrm{f}}{\partial \mathrm{x} \partial \mathrm{y}}\right) \frac{\partial^{2} \mathrm{f}}{\partial \mathrm{y}^{2}}-2 \frac{\partial \mathrm{f}}{\partial \mathrm{x}} \frac{\partial \mathrm{f}}{\partial \mathrm{y}} \frac{\partial^{2} \mathrm{f}}{\partial \mathrm{x} \partial \mathrm{y}}+\left(1+\frac{\partial^{2} \mathrm{f}}{\partial \mathrm{y}^{2}}\right) \frac{\partial^{2} \mathrm{f}}{\partial \mathrm{x}^{2}}}{2\left(1+\frac{\partial^{2} \mathrm{f}}{\partial \mathrm{x} \partial \mathrm{y}}+\frac{\partial^{2} \mathrm{f}}{\partial \mathrm{x}^{2}}+\frac{\partial^{2} \mathrm{f}}{\partial \mathrm{y}^{2}}\right)}
\end{gathered}
$$

Here $\mathrm{f}$ is a real valued function vector in each direction Y,R and P.

$$
\text { Normalized Curvature }(\mathrm{NC})=\frac{\frac{\partial^{2} \mathrm{f}}{\partial \mathrm{x}^{2}} \frac{\partial^{2} \mathrm{f}}{\partial \mathrm{y}^{2}}-\frac{\partial^{2} \mathrm{f}}{\partial \mathrm{x} \partial \mathrm{y}}}{2\left(1+\frac{\partial^{2} \mathrm{f}}{\partial \mathrm{x} \partial \mathrm{y}}+\frac{\partial^{2} \mathrm{f}}{\partial \mathrm{x}^{2}}+\frac{\partial^{2} \mathrm{f}}{\partial \mathrm{y}^{2}}\right)}
$$

Where $f_{x}, f_{y}$ are the first order partial derivatives, $\mathrm{f}_{\mathrm{xy}}, \mathrm{f}_{\mathrm{x}}^{2}, \mathrm{f}_{\mathrm{y}}^{2}$ are the second order partial derivatives . Thus for each point in the 3-dimensional vector space ,partial derivatives are computed to estimate curvatures. These curvatures are used to find the edges in each direction.

Algorithm 4:Ensemble Graph Based Facial Expression Recognition Model

Input : Filtered 3D faces

Output: Expression Recognition and Marking

Procedure:

Step 1: For each dimension $(\mathrm{Y}, \mathrm{P}, \mathrm{R})$ axis

Construct graph model as

$$
\begin{aligned}
& P_{\mathrm{Y}}=\operatorname{ProbChainGraph}(\mathrm{Y}) ; \\
& \mathrm{PG}_{\mathrm{P}}=\operatorname{ProbChainGraph}(\mathrm{P}) ; \\
& \mathrm{PG}_{\mathrm{R}}=\operatorname{ProbChainGraph}(\mathrm{R}) ;
\end{aligned}
$$

Extract $\mathrm{E}\left(\mathrm{PG}_{\mathrm{Y}}\right), \mathrm{E}\left(\mathrm{PG}_{\mathrm{P}}\right), \mathrm{E}\left(\mathrm{PG}_{\mathrm{R}}\right)$ as Edges sets in $\mathrm{Y}, \mathrm{P}, \mathrm{R}$ direction .

End for

Step 2: For each dimension (Y,P,R)

Do

Seg_PG $\mathrm{PG}_{\mathrm{Y}}\left[\mathrm{D}=\right.$ Apply enhanced chaining graph model in $\mathrm{Y}$ direction for $\mathrm{PG}_{\mathrm{Y}}$; Seg_ $\mathrm{PG}_{\mathrm{P}}[]=$ Apply enhanced chaining graph model in $\mathrm{P}$ direction for $\mathrm{PG}_{\mathrm{P}}$; Seg_PG ${ }_{R}[]=$ Apply enhanced chaining graph model in $R$ direction for $\mathrm{PG}_{\mathrm{R}}$;

Done

Let $r_{k}$ be the randomly selected region in each direction.

For each region $r_{i}$ in $\mathrm{SPG}_{\mathrm{Y}}[]$

Do

Find center of region in $\mathrm{Y}$ direction as 


$$
\mathrm{c}_{\mathrm{y}}=\min \left\{\frac{\left|\mathrm{r}_{\mathrm{k}}-\mathrm{r}_{\mathrm{i}+1}\right|}{\left|\mathrm{SPG}_{\mathrm{Y}}[]\right|}\right\} \text { where } \mathrm{i}=\left\{\left\{1,2 \ldots\left|\mathrm{SPG}_{\mathrm{Y}}[]\right|\right\}-\mathrm{k}\right\}
$$

Set $\lambda_{\mathrm{y}}=\mathrm{c}_{\mathrm{y}}$

Compute similarity between the regions along the $\mathrm{Y}$ direction as

$$
\operatorname{Sim}_{-} \mathrm{PG}_{\mathrm{Y}}[]=(1 / 2 \pi) \exp \left(-\left|\mathrm{r}_{\mathrm{k}}-\mathrm{r}_{\mathrm{i}}\right|^{2} / 2\right) * \max \left(\sigma_{\mathrm{i}}^{2}, \sigma_{\mathrm{k}}^{2}\right)
$$

If $\left(\operatorname{Sim} \_\mathrm{PG}_{\mathrm{Y}}[\mathrm{i}]<\lambda_{\mathrm{y}}\right)$

\section{Then}

Find the angular expression computation in the direction $\operatorname{Yaw}(\mathrm{Y})$.

$$
\text { Ang_PG } \mathrm{PG}_{\mathrm{Y}}=\cos ^{-1}\left(\mathrm{E}\left(\mathrm{r}_{\mathrm{k}}\right) \cdot \mathrm{E}\left(\mathrm{r}_{\mathrm{i}}\right) /\left\|\mathrm{E}\left(\mathrm{r}_{\mathrm{k}}\right)\right\| \cdot \| \mathrm{E}\left(\mathrm{r}_{\mathrm{i}} \mid\right) \mid\right)
$$

Where $E\left(r_{k}\right), E\left(r_{i}\right)$ are the edge sets of regions $r_{k}, r_{i}$ in $\mathrm{Y}$ direction.

Mark edge set points in the direction of angle Ang_PG ${ }_{Y}$.

Done

For each region $r_{i}$ in $\mathrm{SPG}_{\mathrm{P}}[]$

Do

Find center of region in $\mathrm{P}$ direction as

$\mathrm{c}_{\mathrm{p}}=\min \left\{\frac{\left|\mathrm{r}_{\mathrm{k}}-\mathrm{r}_{\mathrm{i}+1}\right|}{\left|\mathrm{SPG}_{\mathrm{P}}[]\right|}\right\}$ where $\mathrm{i}=\left\{\left\{1,2 \ldots\left|\mathrm{SPG}_{\mathrm{P}}[]\right|\right\}-\mathrm{k}\right\}$

Set $\lambda_{\mathrm{p}}=\mathrm{c}_{\mathrm{p}}$

Compute similarity between the regions along the $\mathrm{P}$ direction as

$\operatorname{Sim}_{-} \mathrm{PG}_{\mathrm{P}}[]=(1 / 2 \pi) \exp \left(-\left|\mathrm{r}_{\mathrm{k}}-\mathrm{r}_{\mathrm{i}}\right|^{2} / 2\right) * \max \left(\sigma_{\mathrm{i}}^{2}, \sigma_{\mathrm{k}}^{2}\right)$;

If $\left(\operatorname{Sim}_{-} \mathrm{PG}_{\mathrm{P}}[\mathrm{i}]<\lambda_{\mathrm{p}}\right)$

Then

Find the angular expression computation in the direction P.

$$
\text { Ang_PG } \mathrm{P}_{\mathrm{P}}=\cos ^{-1}\left(\mathrm{E}\left(\mathrm{r}_{\mathrm{k}}\right) \cdot \mathrm{E}\left(\mathrm{r}_{\mathrm{i}}\right) /\left\|\mathrm{E}\left(\mathrm{r}_{\mathrm{k}}\right)\right\| \cdot \| \mathrm{E}\left(\mathrm{r}_{\mathrm{i}} \mid\right) \mid\right)
$$

Where $E\left(r_{k}\right), E\left(r_{i}\right)$ are the edge sets of regions $r_{k}, r_{i}$ in $\mathrm{P}$ direction.

Mark edge set points in the direction of angle Ang_PG ${ }_{\mathrm{P}}$.

Done
For each region $r_{i}$ in $\mathrm{SPG}_{\mathrm{R}}[]$

Do

Find center of region in $\mathrm{Y}$ direction as

$\mathrm{c}_{\mathrm{r}}=\min \left\{\frac{\left|\mathrm{r}_{\mathrm{k}}-\mathrm{r}_{\mathrm{i}+1}\right|}{\left|\mathrm{SPG}_{\mathrm{R}}[]\right|}\right\}$ where $\mathrm{i}=\left\{\left\{1,2 \ldots\left|\mathrm{SPG}_{\mathrm{R}}[]\right|\right\}-\mathrm{k}\right\}$

Set $\lambda_{\mathrm{r}}=\mathrm{c}_{\mathrm{r}}$

Compute similarity between the regions along the $\mathrm{R}$ direction as

$\operatorname{Sim}_{-} \mathrm{PG}_{\mathrm{R}}[]=(1 / 2 \pi) \exp \left(-\left|\mathrm{r}_{\mathrm{k}}-\mathrm{r}_{\mathrm{i}}\right|^{2} / 2\right) * \max \left(\sigma_{\mathrm{i}}^{2}, \sigma_{\mathrm{k}}^{2}\right) ;$

If $\left(\operatorname{Sim} \_\mathrm{PG}_{\mathrm{R}}[\mathrm{i}]<\lambda_{\mathrm{p}}\right)$

Then

Find the angular expression computation in the direction $\mathrm{R}$.

$$
\text { Ang_PG }{ }_{\mathrm{R}}=\cos ^{-1}\left(\mathrm{E}\left(\mathrm{r}_{\mathrm{k}}\right) \cdot \mathrm{E}\left(\mathrm{r}_{\mathrm{i}}\right) /\left\|\mathrm{E}\left(\mathrm{r}_{\mathrm{k}}\right)\right\| \cdot \| \mathrm{E}\left(\mathrm{r}_{\mathrm{i}} \mid\right) \mid\right)
$$

Where $E\left(r_{k}\right), E\left(r_{i}\right)$ are the edge sets of regions $r_{k}, r_{i}$ in $\mathrm{R}$ direction.

Mark edge set points in the direction of angle Ang_PG $\mathrm{PG}_{\mathrm{R}}$.

Done

\section{EXPERIMENTAL RESULTS}

The evaluation of the proposed system is carried out using standard performance measures which are of falsepositiverate(fpr), truepositiverate(tpr), accuracy and errorrate, true positives(tp), false positives(fp), true negatives(tn), false negatives(fn). (fp+tn) indicates the total number of pixels represented foreground and background. 2D human pose data sets like CAVIAR (2004), CASIA ACTION (2007) and ETISEO (2005) are used in our experimental study for human pose recognition. A three-dimensional face showing different facial expressions, acquired using a real-time range camera [1]. The database contains 15 3D facial expressions of the same face, represented as a XYZ(.vert) and ASCII text file format.

$$
\begin{gathered}
f p r=\frac{f p}{f p+t n} ; t p r=\frac{t p}{t p+f n} ; \\
\text { accuracy }=\frac{t p}{t p+f n+f p+t n} ; \\
\text { errorrate }=1-\text { accuracy }
\end{gathered}
$$




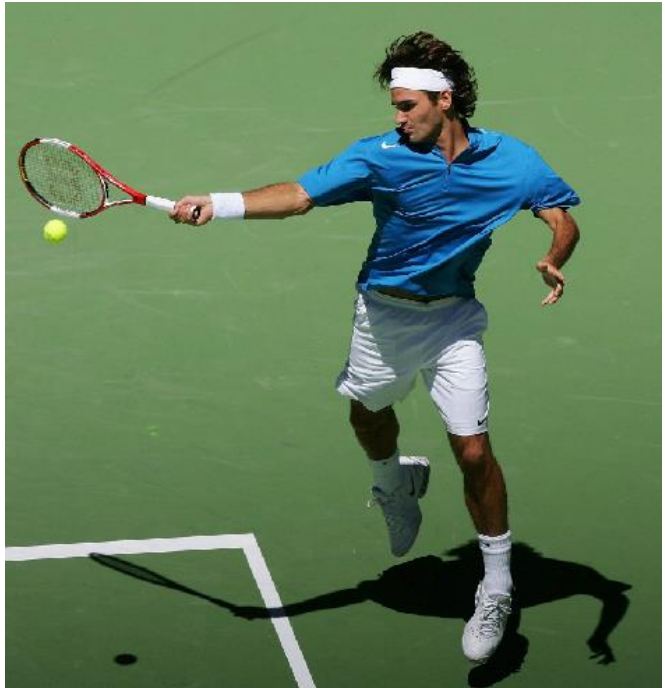

Fig.7. Input Image

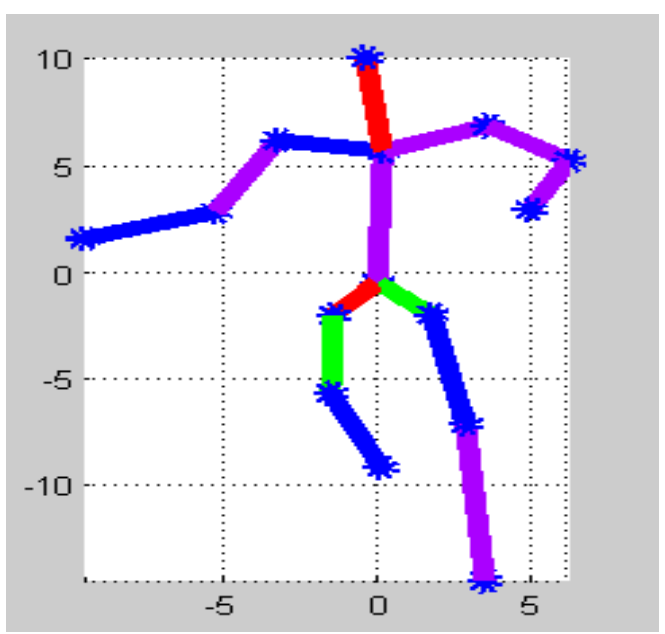

Fig.8. 2D action recognition

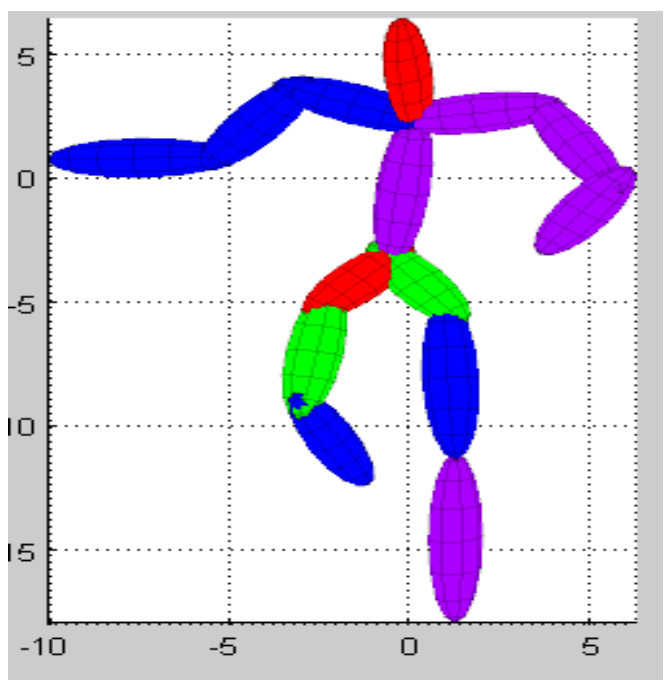

Fig.9. 2D X-Y Directional View

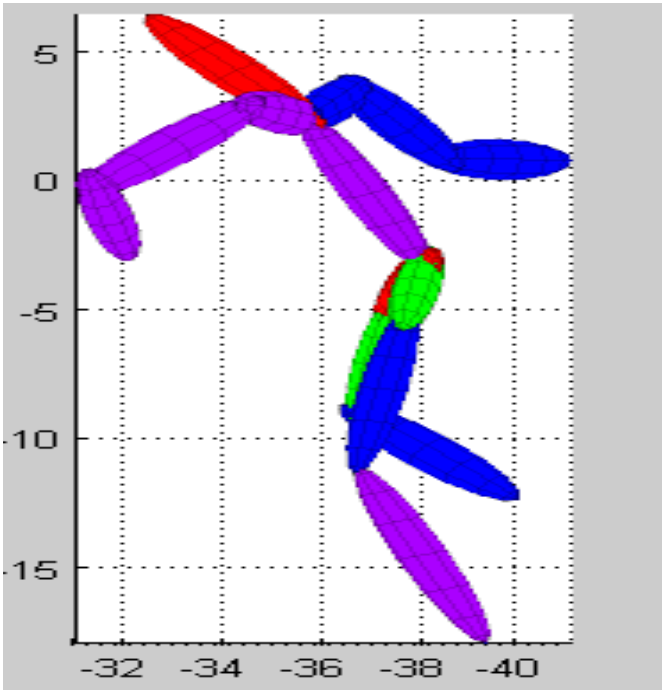

Fig.10. 2D Y-Z Directional View

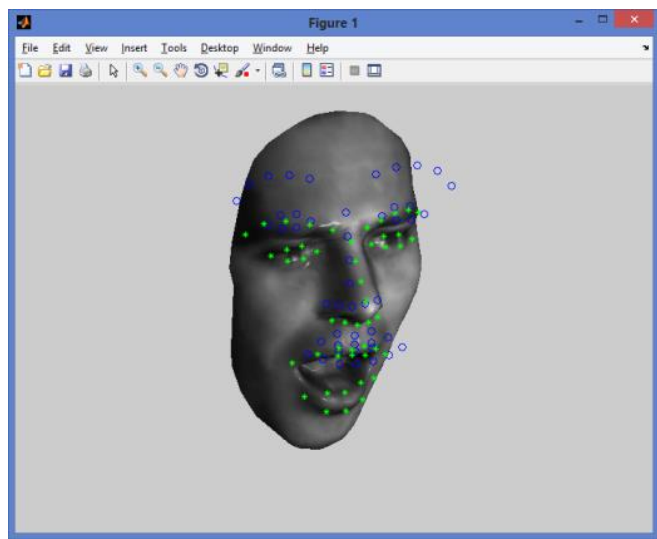

Fig.11. Traditional 3D Facial Pose estimation using Edge based Model

Fig. 11. Shows the 3D facial expression evaluated using the proposed graph based edge detection model. From the figure, it is observed that green marks are identified using our proposed model as facial three dimensional expressions and blue marks indicates the traditional otsu's based edge detection model.

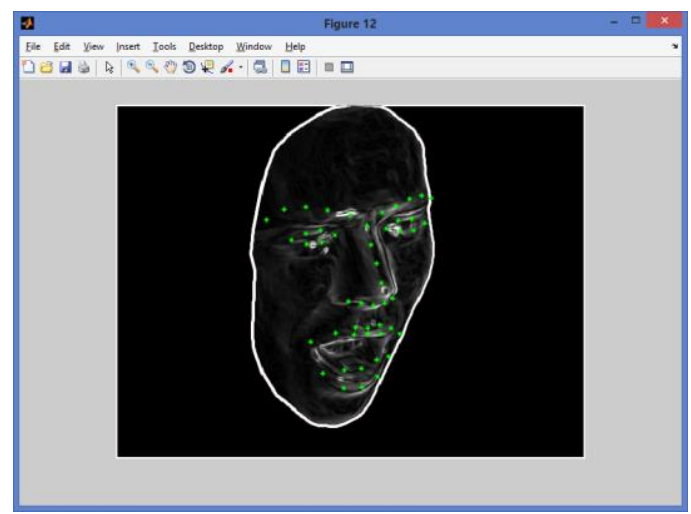

Fig.12. Proposed Ensemble based 3D Facial Pose Estimation Model 
Table 1. True Positive rate of the existing and proposed model on $2 \mathrm{D}$ video sequences

\begin{tabular}{|c|c|c|}
\hline 2D-Dataset & Avg TPR(EHPM) & $\begin{array}{c}\text { Proposed } \\
\text { ECGPM }\end{array}$ \\
\hline CAVIAR & 0.97163 & 0.97935 \\
\hline CASIA & 0.96744 & 0.98353 \\
\hline ETISEO & 0.97824 & 0.98024 \\
\hline
\end{tabular}

Table.1. shows the average true positive rate of the enhanced hybrid probabilistic model and the proposed ensemble based chaining graph probabilistic model. By observing the table it is clear that the proposed technique can perform better true positive rate compare to the existing technique.

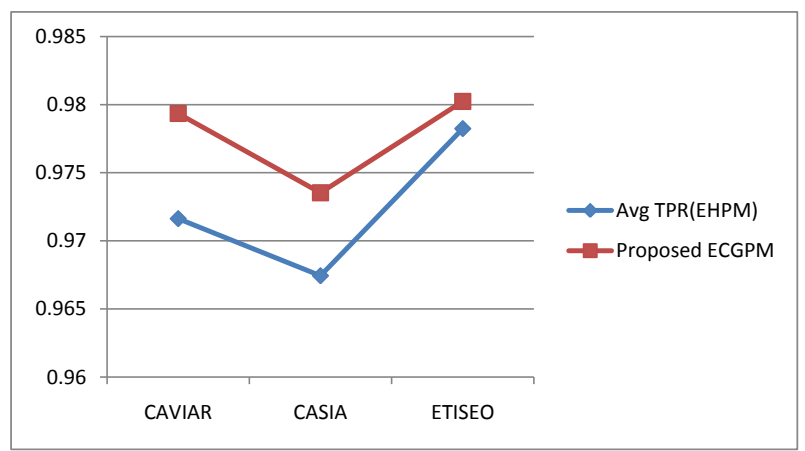

Fig.13. Avg True Positive Rate of the existing and proposed model

Fig.13., shows the average true positive rate of the enhanced hybrid probabilistic model and the proposed ensemble based chaining graph probabilistic model. By observing the table it is clear that the proposed technique can perform better true positive rate compare to the existing technique.

Table 2. RunTime comparison of proposed and existing models on 2Dpose estimation.

\begin{tabular}{|c|c|}
\hline Algorithm & RunTime(Secs) \\
\hline Texture based Pose Estimation & 163 \\
\hline JBPCG & 154 \\
\hline EJBPCG & 139 \\
\hline Proposed Model & 125 \\
\hline
\end{tabular}

Table 2. describes the runtime comparison of proposed and existing model on 2D-videos and 3D-facial expression evaluation. From this table, it is observed that proposed model has less runtime compared to traditional models.

Table 3. RunTime comparison of proposed and existing models on 3Dfacial expression evaluation

\begin{tabular}{|c|c|}
\hline Algorithm & RunTime(Secs) \\
\hline Texture based Pose Estimation & NA \\
\hline JBPCG & 89 \\
\hline EJBPCG & 78 \\
\hline Proposed Model & 64 \\
\hline
\end{tabular}

Table 3. describes the runtime comparison of proposed and existing model on 3D-facial expression evaluation. From this table, it is observed that proposed model has less runtime compared to traditional models.

Table 4. True Positive Rate of the existing and proposed model on 3D facial pose estimation

\begin{tabular}{|c|c|c|}
\hline 3D-Dataset & Avg TPR(EHPM) & Proposed ECGPM \\
\hline Image 1 & 0.945 & 0.9674 \\
\hline Image 2 & 0.954 & 0.9598 \\
\hline Image 3 & 0.949 & 0.9643 \\
\hline Image 4 & 0.956 & 0.9604 \\
\hline Image 5 & 0.925 & 0.9453 \\
\hline
\end{tabular}

Table 4. Shows the average true positive rate of the enhanced hybrid probabilistic model and the proposed ensemble based chaining graph probabilistic model. By observing the table it is clear that the proposed technique can perform better true positive rate compare to the existing technique.

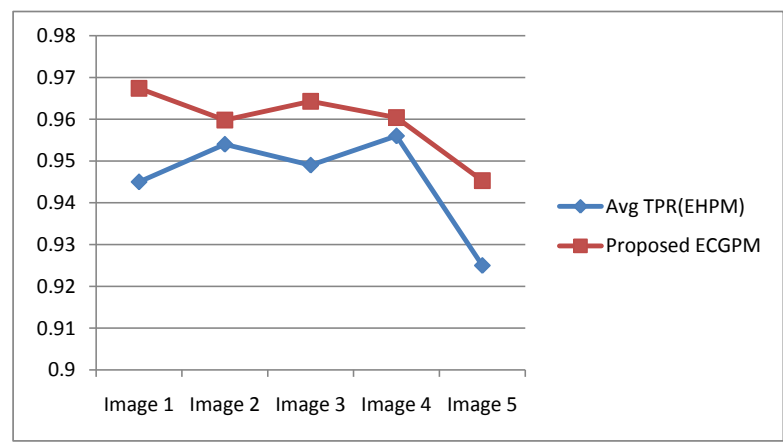

Fig.14. True Positive Rate of the existing and proposed model on 3D facial pose estimation

Fig.14, shows the average true positive rate of the enhanced hybrid probabilistic model and the proposed ensemble based chaining graph probabilistic model. By observing the table it is clear that the proposed technique can perform better true positive rate compare to the existing technique.

\section{CONCLUSION}

In this paper, we have proposed a novel $2 \mathrm{D} / 3 \mathrm{D}$ joint ensemble chaining graph model for pose detection. Traditional directed and undirected graphical models such as Bayesian Markov model, conditional random field have limitations with arbitrary pose estimation in 2D/3D images using the joint probabilistic model. To overcome these issues, we introduce an ensemble chaining graph model to estimate arbitrary human poses in 2D video sequences and facial expression evaluation in 3D images. This system has three main hybrid algorithms, namely $2 \mathrm{D} / 3 \mathrm{D}$ human pose pre-processing algorithm, ensemble graph chaining segmented model on 2D/3D video sequence pose estimation and $3 \mathrm{D}$ ensemble facial expression detection algorithm. The experimental results 
on public benchmarks 2D/3D datasets show that our model is more efficient in solving arbitrary human pose estimation problem. Also, this model has high true positive rate, low false detection rate compared to traditional joint human pose detection models. In future, this work can be extended to detect various 3D motion detection using a hybrid multi-objective based probabilistic graphical segmentation model.

\section{REFERENCES}

[1] http://tosca.cs.technion.ac.il/data/face.zip

[2] Y. Freund and R. E. Schapire. A decision-theoretic generalization on on-line learning and an application to boosting. Journal of Computer and System Sciences, 55(1):119-139, 1997.

[3] A. H. Gee and R. Cipolla. Determine the gaze of faces in images. Image and Vision Computing, 12(10):639-647, 1994.

[4] C. Dharmagunawardhana, S. Mahmoodi, M. Bennett and M. Niranjan, "Gaussian Markov random field based improved texture descriptor for image segmentation", Image and Vision Computing, vol. 32, no. 11, pp. 884-895, 2014.

[5] B. Sun and J. He, "Discriminative dictionary based representation and classification of image texture", Sixth International Conference on Digital Image Processing (ICDIP 2014), 2014.

[6] N. Wang, "Color Image Edge Detection Based on Cube Similarity", 2015 IEEE International Conference on Computational Intelligence \& Communication Technology, 2015.

[7] X. Zhang and C. Liu, "An ideal image edge detection scheme", Multidimensional Systems and Signal Processing, vol. 25, no. 4, pp. 659-681, 2013.

[8] K. Zhang and K. Lam, "A Level Set Approach to Image Segmentation With Intensity Inhomogeneity", IEEE Trans. Cybern., vol. 46, no. 2, pp. 546-557, 2016.

[9] E. Murphy Chutorian and M. Manubhai Trivedi, Head Pose Estimation in Computer: A Survey, IEEE Transaction on Pattern Analysis and Machine Intelligence, Vol. 31. No.4, pp. 607627, 2009.

[10] G. Fanelli, J. Gall, and L. Van Gool, "Real time head pose estimation with random regression forests," in CVPR, 2011.

[11] K.I. Chang, K.W. Bowyer, P.J. Flynn, "An evaluation of multi-model 2D+3D biometrics", IEEE Transactions on Pattern Analysis and Machine Intelligence 27 (4) (2005) 619-624.

[12] P. S. Hiremath, Manjunatha Hiremath,"Depth and Intensity Gabor Features Based 3D Face Recognition Using Symbolic LDA and AdaBoost", IJIGSP, vol.6, no.1, pp.32-39, 2014.DOI: 10.5815/ijigsp.2014.01.05.

[13] K.I. Chang, K.W. Bowyer, P.J. Flynn, "Multiple nose region matching for $3 \mathrm{D}$ face recognition under varying facial expression", IEEE Transactions on Pattern Analysis and Machine Intelligence 28 (10) (2006) 1695-1700.

[14] P. S. Hiremath, Manjunatha Hiremath,"3D Face Recognition based on Radon Transform, PCA, LDA using KNN and SVM", IJIGSP, vol.6, no.7, pp.36-43, 2014.DOI: 10.5815/ijigsp.2014.07.05.

[15] Kevin W. Bowyer, Kyong Chang, Patrick Flynn, "A survey of approaches and challenges in 3D and multimodal 3D + 2D face recognition", Computer Vision and Image Understanding 101, 1-15 (2006).

\section{Authors' Profiles}

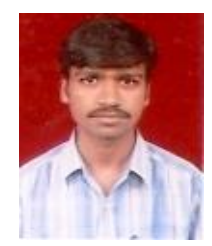

D.Ratna kishor received B.Tech degree in Computer Science and Engineering from JNTU Hyderabad,A.P India and M.Tech degree in in Computer Science from Jawaharlal Nehru Technological University Hyderabad(JNTUH) Hyderabad,A.P,india. He is now pursuing her Ph.D degree from JNT University Anantapur.He is now working as a Asst.Prof in department of Computer Science and Engineering, Dhanekula institute of engg \& tech, A.P.

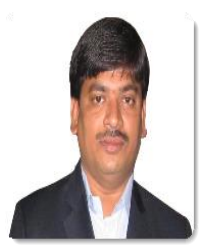

M. Chandra Mohan received B.E. (EEE) degree from Osmania University in 1994. He worked as Assistant Engineer in AP State Electricity Board (APSEB) for 7 years (19942001). He completed his M.Tech. (CS\&E) from Osmania University in 2000 . He is working in JNT University Hyderabad since 2001. Presently he is working as an Professor in Dept of CS\&E in JNTUH College of Engineering Hyderabad, JNT University Hyderabad. He is the recipient of 3 Gold Medals from Osmania University at the graduate level by securing University first rank. He completed his Ph.D in 2010 from JNTU Hyderabad in Computer Science \& Engineering. He has more than 30 publications in various national and international journals and conferences.

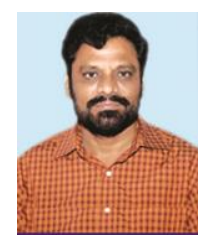

Dr. A.Ananda Rao received B.Tech degree in Computer Science and Engineering from University of Hyderabad, A.P, India and M.Tech degree in Artificial Intelligence and Robotics from University of Hyderabad, A.P, India. He received $\mathrm{Ph} . \mathrm{D}$ degree from Indian Institute of Technology Madras, Chennai, India. He is Professor in Computer Science \& Engineering and currently discharging his duties as Director Academic \& Planning, JNT University Anantapur, Ananthapuramu, A.P, India. Dr. Rao has more than 100 publications in various National and International Journals/Conferences and authored three text books. He received one Best Research Paper award, one Best Paper award for his papers. He received many awards such as Best Teacher Award form Govt. of Andhra Pradesh in 2014, Best Computer Science Engineering Teacher award from ISTE, AP Chapter in 2013. His main research interest includes Software Engineering and Databases. 
How to cite this paper: D.Ratna kishore, M. Chandra Mohan, Akepogu. Ananda Rao,"A Novel Joint Chaining Graph Model for Human Pose Estimation on 2D Action Videos and Facial Pose Estimation on 3D Images", International Journal of Image, Graphics and Signal Processing(IJIGSP), Vol.9, No.3, pp.21-32, 2017.DOI: 10.5815/ijigsp.2017.03.03 